\title{
Synthesis of Graphene Supported Nickel and Cobalt Nanoparticles and Their Applications for Methanol Oxidation in Alkaline Medium
}

\author{
Muhammad Irfan Raza*, Ehtsham Sarwar*, Naseem Iqbal* and Safeer Ahmed**
}

\begin{abstract}
In various applications fuel cells are considered very attractive devices to obtain clean electrical energy directly from the combustion of chemical fuel. During the last decade much attention is given to the direct methanol fuel cells (DMFCs) because methanol is inexpensive, easily available and transported liquid fuel. Mostly platinum is employed for the electro-oxidation of methanol as an anodic material however higher economic costs and poor oxidation kinetics are the main problems of platinum based catalyst. In this paper we reported the synthesis of graphene oxide (GO) by modified Hummer's method and synthesis of graphene supported cobalt and nickel nanoparticles by simple chemical solution synthesis method. The synthesized materials were characterized by X-ray diffraction (XRD), scanning electron microscopy (SEM) and EDX. The electrocatalytic properties of the synthesized catalyst for methanol oxidation were investigated by cyclic voltammetry.
\end{abstract}

Keywords - Graphene, Graphene oxide, Direct methanol fuel cells, Nanocomposites, Electrocatalysts, Methanol oxidation.

\section{INTRODUCTION}

Under the global sustainability prospective, sustainable development involves four key factors in terms of social, environmental, economic and energy resources sustainability. Therefore energy derives the global economy and is considered as key element for the climate and the sustainable development. Sustainable development requires sustainable supply of clean and cheap sources of energy that do not have adverse social and environmental impacts[1][2]. Globally, about $87 \%$ of total energy is produced by fossil fuels about $6 \%$ is generated in nuclear power plants and only $7 \%$ comes from renewable resources (hydro, wind, solar, geothermal and biofuel). Unfortunately, this planet has limited amount of fossil and nuclear energy resource and at the same time generation of energy can contribute to degradation of local environment such as global warming, acid rain and ozone layer depletion ultimately the climate change[3]. In order to overcome all these critical challenges of energy security and environmental degradation attempts have been made in the development

U.S.-Pakistan Centre for Advanced Studies in Energy, NUST, Sector H-12, Islamabad 44400

** Department of chemistry, Quaid-i-Azam, University, Islamabad, 45320, Pakistan

Corresponding Author; Ehtsham Sarwar , U.S.-Pakistan Centre for Advanced Studies in Energy, NUSTil: of advance, affordable and environment friendly energy harnessing technologies.

In this regard innovative energy technologies has got considerable attention during the last few decades. Among them fuel cell is considered as promising power generation technology both for mobile and stationary applications[4].

Success of DMFC to be competitive as viable energy source in the energy market depends upon the cost, efficiency and durability of the electrocatalyst. At present, most of the fuel cells (low temperature fuel cell) use Ptbased catalyst. Due to these expensive electrocatalyst manufacturing cost of the fuel cell is increased which greatly affects the successful commercialization[5]. Beside these most of the Pt-based electrocatalyst face catalyst poisoning. Consequently researchers are trying to develop a low cost, reliable and efficient electrocatalyst to replace Pt-base catalyst[6].

So far significant numbers of studies have been performed by involving different techniques for the electrooxidation of lower molecular weight organic compounds such as $\mathrm{CH}_{3} \mathrm{OH}, \mathrm{HCOOH}$, and $\mathrm{HCHO}$. Results from these investigations reveals that at low temperature electrooxidation of these organic compounds need the presence of Ptbased catalyst[7]. Two key steps dehydrogenation and chemisorption of $\mathrm{CO}$ are involved during the electro-oxidation of these compounds under Pt-based catalyst. A strongly adsorbed intermediate species (like $\mathrm{CO}, \mathrm{CHO}$ ) are produced during all these reactions. These types of species like CO, $\mathrm{CHO}$ are adsorbed on the surface of the catalyst and cause for catalyst poisoning[8]. The poisoning effect of the Pt catalyst significantly decreases power density and fuel consumption efficiency of the cell. To inhibit the poisoning effect and to increase the electro-oxidation rate by an order of three to four times a lot of research have been carried out to replace the $\mathrm{Pt}$ catalyst[9]. Most suitable results have been achieved by alloying the $\mathrm{Pt}$ with $\mathrm{Ru}$ and $\mathrm{Sn}$ for the complete oxidation of methanol and $\mathrm{CO}$ into $\mathrm{CO}_{2}[10]$. In recent years research is focused to develop non noble metal electrocatalysts such as $\mathrm{Co}, \mathrm{Ni}, \mathrm{Pb}$, and $\mathrm{W}$ to replace platinum an expensive metal. However above transition metals show poor oxidation kinetics. By using high conductive material as a support like graphene, carbon nanotubes (CNTs) and carbon nanofibers, 
electrocatalytic efficiency of these metals can be improved. Compared with other carbon support material graphene is an excellent electrode material because of its lower manufacturing cost, high surface area, and exceptional conductivity[11]. Therefore, graphene can be used as a catalytic support material to enhance the electrochemical activity of catalyst particles for methanol and ethanol oxidation[12]. In our present work we synthesized $\mathrm{Ni} /$ graphene and $\mathrm{Co} / g r a p h e n e$ nanocomposites by a facile, economic and environmental friendly method. Furthermore, the synthesized materials were characterized by XRD, SEM and EDX. The electrochemical activity of the synthesized electrocatalyst towards methanol oxidation was characterized by cyclic voltammetry technique.

\section{II.EXPERIMENTAL}

\section{A. Material and Reagents}

Graphite Powder $(99.99 \%)$ of particle size $45 \mu \mathrm{m}$, H2SO4 (98\%), NaNO3 (99\%), KMnO4 (99\%), H2O2 (30\%), NiSO4.6H2O, $\mathrm{Co}(\mathrm{NO} 3) 2.6 \mathrm{H} 2 \mathrm{O}$, Ethylene Glycol, NH3 were purchased from Sigma Aldrich (USA). All the reagents were of analytical grades and used without further purification.

\section{B. Preparation of Graphene Oxide (GO)}

Graphene oxide (GO) was synthesized from graphite powder by modified Hummer's method[13]. In the typical procedure $1 \mathrm{~g}$ of graphite powder(99.99\%) was obtained and mixed with $25 \mathrm{ml} 98 \%$ sulphuric acid (H2SO4), and $1 \mathrm{~g}$ of sodium nitrate $(\mathrm{NaNO} 3)$ taken in an ice bath for three hours followed by the gradual addition of $5 \mathrm{~g}$ of $\mathrm{KMnO} 4$. The reaction was kept under vigorous stirring at $30{ }^{\circ} \mathrm{C}$. Then slow addition of $100 \mathrm{ml}$ water is performed. After this the obtained product was placed at $85^{\circ} \mathrm{C}$ for two hours, then reaction was treated with $10 \mathrm{ml}$ hydrogen peroxide $(\mathrm{H} 2 \mathrm{O} 230 \%)$. Finally, the obtained yellowish cake was centrifuged in order to get pure product where solution was washed several times with distilled water and ethanol.

\section{C.Preparation of Ni/Co Graphene Nano composites}

In the present work deposition of nickel nanoparticles on graphene was obtained by using simple solution synthesis method[14]. In this method we used ethylene glycol as a solvent and hydrazine hydrate as a reducing agent. In the typical procedure, $262.8 \mathrm{mg}$ of nickel sulfate hexa-hydrate (NiSO4.6H2O), and $100 \mathrm{mg}$ of graphene oxide that was obtained from graphite powder oxidation by a modified Hummer's method. Nickel salt as metal precursor and graphene oxide were added in a $100 \mathrm{ml}$ flask having $60 \mathrm{ml}$ ethylene glycol that was used as a solvent. After that the mixture was ultrasonicated for an hour, then stirring was performed for 24 hours at ambient temperature. Then ammonia solution was added into the solution while constant stirring in order to gradually decrease the $\mathrm{pH}$ of the solution to 10.5. After that $1.0 \mathrm{ml}$ hydrazine hydrate was added as a reducing agent into the above solution and the reduction reaction was performed under constant stirring at $85^{\circ} \mathrm{C}$ for 12 hours. The obtained product was then filtered in order to get pure product, washing was performed various times with distilled water and ethanol and dried in a vacuum desiccator at room temperature. Same procedure is adopted for the

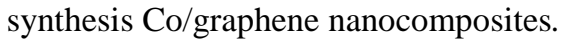

\section{D.SEM /XRD Characterization}

In order to investigate the phase, crystal structure and lattice parameter of the prepared nanocomposites, X-ray powder diffractometer (STOE Germany) with computer interface was used having radiation source $\mathrm{Cu} \mathrm{K} \square$ at $\lambda=1.5418 \AA$. The scan angle $2 \square$ was selected $0^{\circ}-80^{\circ}$ with step size $0.04 / \mathrm{sec}$.

To study the surface morphology of the synthesized catalyst Scanning Electron Microscope (HITACHI SU- 1500) with $50 \mu \mathrm{m}$ magnification was used and voltage was kept up-to 15 $\mathrm{kV}$.

\section{E. Electrochemical Measurements}

All the electrochemical measurements were performed by using AUTOLAB 4.9 Potentiostat connected to PC. We used $\mathrm{Ag} / \mathrm{AgCl}$ electrode as reference electrode in this work. The counter electrode was a $\mathrm{Pt}$ wire $1 \mathrm{~mm}$ thick and $12 \mathrm{~cm}$ long. $1 \mathrm{M}$ $\mathrm{KOH}$ solution was used as a supporting electrolyte with $3 \mathrm{M}$ methanol concentration in a conventional three-electrode Pyrex glass electrochemical cell. The electrochemical characterization of the prepared $\mathrm{Ni} /$ graphene and $\mathrm{Co} /$ graphene nano-composites was done by using cyclic voltammetry technique. In the present work, glassy carbon electrode with active area $0.071 \mathrm{~cm} 2$ was modified as working electrode. The working electrode was first washed and cleaned with distilled water than polished with alumina slurry. For the modification of working electrode a homogenous slurry of the functional material was prepared by mixing $2 \mathrm{mg}$ fine powder of the active material, $20 \mu \mathrm{l}$ of nafion solution and $400 \mu \mathrm{l}$ of isopropanol. After sonicating the slurry for $30 \mathrm{~min}$ at room temperature, $15 \mu \mathrm{l}$ from the prepared slurry was poured on the active surface of the glassy carbon electrode which was then air dried at room temperature. The obtained current densities were normalized based on the utilized surface area of the modified glassy carbon electrode. The response of peak current was checked at various scan rates ranging from $25 \mathrm{mVs}-1$ to $100 \mathrm{mVs}-1$ at starting potential starting from $0 \mathrm{mV}$ to $1800 \mathrm{mV}$ positive vertex potential.

\section{RESUlt AND DISCUSSION}

To investigate the successful synthesis of nano-composites characterization was done by performing $\mathrm{X}$ - ray diffraction and scanning electron microscopy. XRD pattern of graphene oxide (GO) is shown in the figure $1(\mathrm{a})$. The characteristic peak is obtained at $2 \square$ of $11.5^{\circ}$ clearly indicates the synthesis of graphene oxide, which shows that effective oxidation of graphite leads to the formation of graphene oxide. The XRD pattern of prepared Co/graphene nanocomposites is shown in the Fig.1. (b) The typical reflection peak at $2 \square=22.3^{\circ}$ indicates the formation of graphene by the reduction process of graphene oxide. On the other hand, the diffraction bands obtained at $2 \square=37.4^{\circ}, 44.3^{\circ}$ and $63.2^{\circ}, 76.1^{\circ}$ can be assigned to the characteristic of face center cubic (fcc) structure of Co 
nanoparticles.

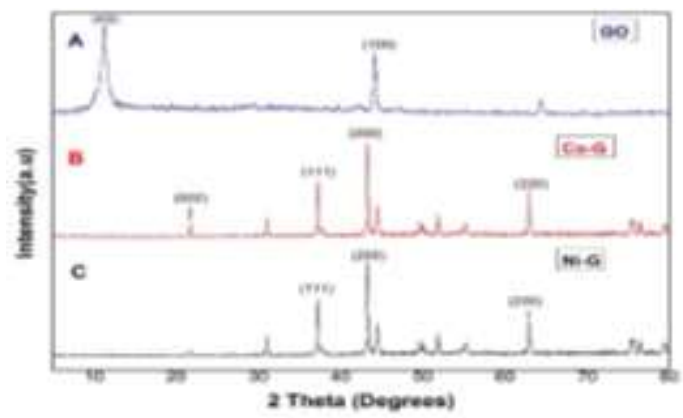

Fig 1. (a) XRD pattern of graphene oxide (b) Co/graphene (c) $\mathrm{Ni} /$ graphene

The XRD display of $\mathrm{Ni} /$ graphene nanocomposites is shown in the Fig.1c. The typical reflection peak at $2 \square=22.3^{\circ}$ indicates the formation of graphene which was obtained by the reduction process of graphene oxide. On the other hand, the diffraction bands obtained at $2 \square=37.4^{\circ}, 44.3^{\circ}$ and $63.2^{\circ}, 76.01^{\circ}$ can be assigned to the characteristic of face center cubic (fcc) structure of Ni nanoparticles. Cobalt and Nickel have almost similar crystal structure under normal conditions due to closer atomic numbers and molecular weights. That is the reason XRD patterns of $\mathrm{Ni}$ and Co are almost similar.

The morphologies of the synthesized materials were characterized by scanning electron microscopy (SEM). Figure.2 (a) represents the low magnification SEM image of graphene oxide from this image the morphology of the secondary particles can be clearly visualized. There might be the chance of agglomeration among the particles. Fig 2 (b) represents the high magnification SEM image of graphene oxide. From this image it can be seen that most of the graphite oxide has been successfully exfoliated to wavy type ultrathin structure of graphene oxide sheets.
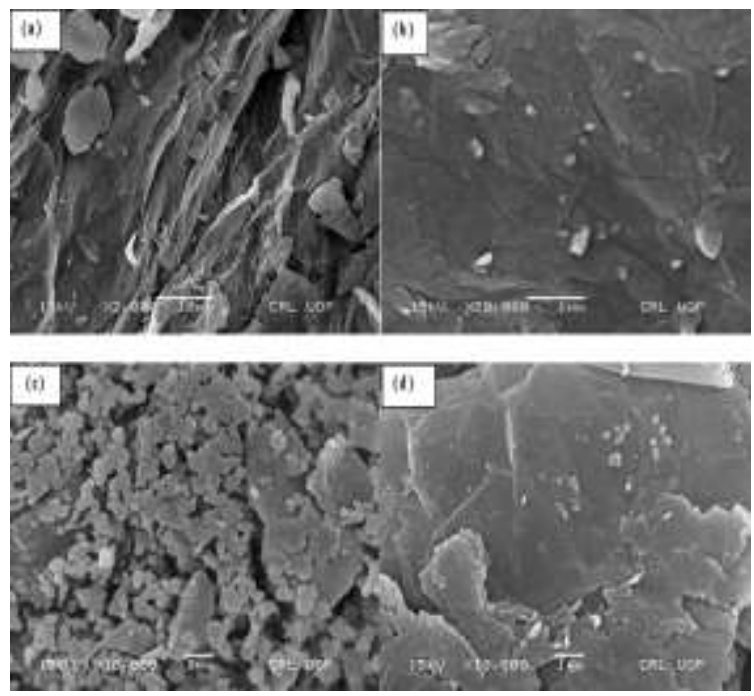

Fig2. SEM image of the (a) graphene sheets (b) Ni/graphene and (c) Co/graphene

Fig. 2c represents the SEM image of $\mathrm{Ni} /$ graphene nanocomposites which reveals that $\mathrm{Ni}$ nanoparticles are uniformly dispersed on graphene sheets. Fig. 2 d represents the randomly dispersed Co nanoparticles on graphene sheets

Figure 3 shows the EDX analysis of Ni/graphene and Co/graphene nanocomposites catalysts. The EDX analysis in the fig 3 a shows that
$\mathrm{Co} /$ graphene nanocomposites catalysts was composed of $\mathrm{C}, \mathrm{O}$ and Co elements. Figure $4 \mathrm{~b}$ represents the EDX spectrum of $\mathrm{Ni} /$ graphene catalysts which reveals that $\mathrm{Ni} /$ graphene nanocomposites catalysts was composed of $\mathrm{C}, \mathrm{O}$ and $\mathrm{Ni}$ elements.

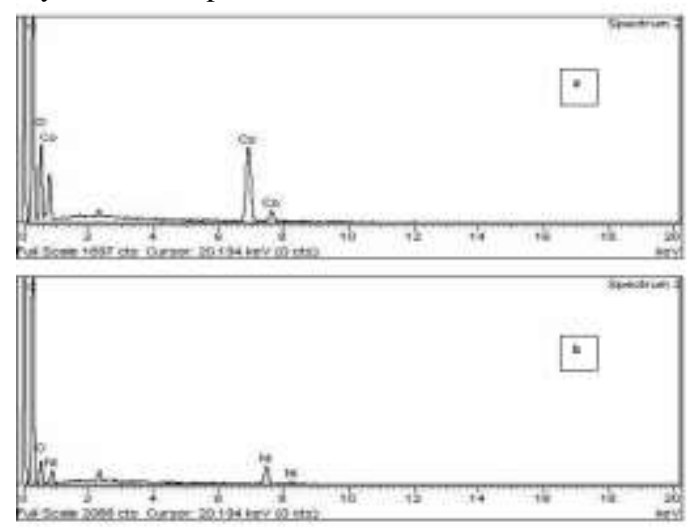

Fig 3. EDX analysis of (a) Co/graphene (b) Ni/ graphene

The presence of carbon peaks in both the samples represents the formation of graphene sheets and small oxygen peak reveals that graphene also contains small quantity of carboxyl and hydroxyl groups.

\subsection{Cyclic Voltammetry}

In order to investigate electrochemical behavior of prepared $\mathrm{Ni}$ / graphene and $\mathrm{Co}$ /graphene cyclic voltammogram was recorded on modified glassy carbon electrode as a working electrode. The cyclic voltammogram $(\mathrm{CVs})$ of $\mathrm{Ni} /$ graphene and $\mathrm{Co}$ /graphene nano-composites for methanol oxidation was done in alkaline medium. For the comparison cyclic voltammogram of bare $\mathrm{GCE}, \mathrm{Ni} /$ graphene and $\mathrm{Co} /$ graphene were recorded under same conditions at a scan rate of 100 $\mathrm{mVs}-1,1 \mathrm{M} \mathrm{KOH}$ and $3 \mathrm{M}$ methanol concentration.

Interestingly it can be seen in the Figure.4 Co/graphene modified electrode showed higher current density during forward scan as compared to bare GCE and Ni/ Graphene electrode.

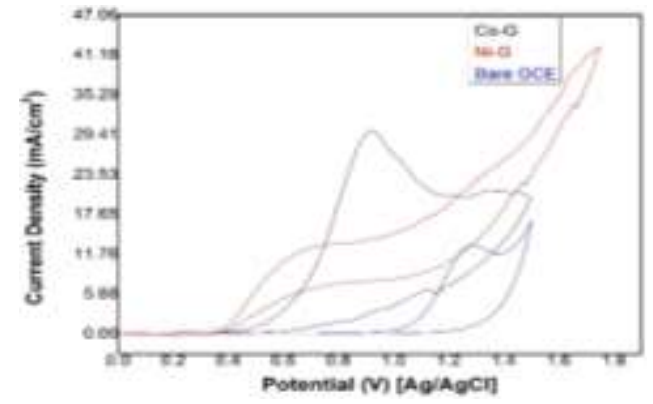

Fig 4. Cyclic voltammogram of bare GCE, graphene supported $\mathrm{Ni}$ and Co nanoparticles at a scan rate of $100 \mathrm{mVs}-1$ at ambient temperature

The anodic peak current observed for the period of forward scan was due to the electro-oxidation of methanol into intermediate species and further complete oxidation of these species into $\mathrm{CO} 2$, $\mathrm{H} 2 \mathrm{O}$ and electrons. During the reverse scan, oxidation peak is obtained due to the remaining species that was not fully oxidized during the forward scan. The cyclic voltammogram of bare GCE electrode, $\mathrm{Ni} /$ graphene and $\mathrm{Co} /$ graphene nanocomposites are shown in the fig. 04. 


\section{CONCLUSION}

$\mathrm{Ni}$ /graphene and $\mathrm{Co} /$ graphene nanocomposites have been synthesized by using chemical reduction method from metal salts and graphene oxide, which was already prepared by the oxidation of graphite powder by using modified Hummer's method. The electrocatalytic properties of these synthesized nanocomposites for methanol oxidation were tested in alkaline medium. It has been observed that $\mathrm{Co} /$ graphene nanocomposites showed higher electrochemical activity as compared to Ni/graphene. However, comparing the current densities of these nanocomposites is far less than Pt/ graphene which is still unbeatable as electrocatalyst for methanol oxidation reaction. Electrochemical stability and potential towards oxygen reduction reaction of these nanocomposites still need further investigations.

\section{References}

[1] I. Dincer, "Environmental and sustainability aspects of hydrogen and fuel cell systems," no. August 2006, pp. 29-55, 2007.

[2] I. Transactions, E. Conversion, and S. Univer, "Environmental Impacts of Electricity Generation: A Global Perspective Environmental Impacts of Electricity Generation :," no. September, 2016.

[3] B. K. Bose, "Energy, Environmental Pollution, and the Impact of Power Electronics," no. March, pp. 6-17, 2010.

[4] O. Z. Sharaf and M. F. Orhan, "An overview of fuel cell technology: Fundamentals and applications," Renew. Sustain. Energy Rev., vol. 32, pp. 810-853, 2014.

[5] H. Liu, C. Song, L. Zhang, and J. Zhang, "A review of anode catalysis in the direct methanol fuel cell," no. April, 2006.

[6] N. A. M. Barakat, M. Motlak, B. Kim, A. G. El-deen, S. S. Al-deyab, and A. M. Hamza, "Journal of Molecular Catalysis A : Chemical Carbon nanofibers doped by Ni x Co $1-\mathrm{x}$ alloy nanoparticles as effective and stable non precious electrocatalyst for methanol oxidation in alkaline media," "Journal Mol. Catal. A, Chem., vol. 394, pp. 177-187, 2014.

[7] N. A. M. Barakat, M. Motlak, A. A. Elzatahry, K. Abdelrazek, and E. A. M. Abdelghani, "ScienceDirect Ni x Co 1 L x alloy nanoparticledoped carbon nanofibers as effective non-precious catalyst for ethanol oxidation," Int. J. Hydrogen Energy, vol. 39, no. 1, pp. 305-316, 2013.

[8] A. S. Aricò, S. Srinivasan, and V. Antonucci, "DMFCs: From Fundamental Aspects to Technology Development," no. 2, pp. 133-161, 2001.

[9] P. O. Box and C. Ch, "Shell Research Limited, Thornton Research Centre, P.O. Box 1, Chester CH1 3SH, England," vol. 118, 1981.

[10] E. V. Spinacé, M. Linardi, and A. O. Neto, "Co-catalytic effect of nickel in the electro-oxidation of ethanol on binary Pt-Sn electrocatalysts," Electrochem. commun., vol. 7, no. 4, pp. 365-369, 2005.

[11] W. Gao, "The chemistry of graphene oxide," Graphene Oxide Reduct. Recipes, Spectrosc. Appl., pp. 61-95, 2015.

[12] Z. Wang, Y. Du, F. Zhang, Z. Zheng, Y. Zhang, and C. Wang, "High electrocatalytic activity of non-noble $\mathrm{Ni}$-Co/graphene catalyst for direct ethanol fuel cells.," Sect. Title Electrochem. Radiational, Therm. Energy Technol., vol. 17, no. 1, pp. 99-107, 2013.

[13] D. C. Marcano, D. V Kosynkin, J. M. Berlin, A. Sinitskii, Z. Sun, A. Slesarev, L. B. Alemany, W. Lu, and J. M. Tour, "Improved Synthesis of Graphene Oxide," vol. 4, no. 8.

[14] N. A. M. Barakat and M. Motlak, "Applied Catalysis B : Environmental Co x Ni y -decorated graphene as novel, stable and super effective nonprecious electro-catalyst for methanol oxidation," "Applied Catal. B, Environ., vol. 154-155, pp. 221-231, 2014. 